\title{
Los procesos metarreflexivos en la formación inicial del profesorado
}

\author{
Meta-reflexive processes in pre-service teacher education
}

Julio Cabrera, Mańa Inés Rodríguez Vida y Elsa Meinardi

GDB - Grupo de Didáctica de la Biología, Centro de Formación e Investigación en Enseñanza de las Ciencias (CEFIEC), Facultad de Ciencias Exactas y Naturales, Universidad de Buenos Aires. Ciudad Autónoma de Buenos Aires, Argentina. emeinardi@fibertel.comar

\section{Resumen}

En este trabajo se analiza la producción de prácticas docentes en escuela media, a partir del marco teórico del Modelo Cognitivo de Ciencia Escolar.

La indagación formó parte de la tesis final de la formación de profesores del primer autor, quien analizó sus propias prácticas de dase y las de otros docentes. El trabajo permitió establecer el grado de coherencia entre las formulaciones teóricas y las prácticas que construyen los alumnos del profesorado y también determinar la capacidad de análisis y reflexión que desarrollan durante su formación profesional.

El análisis de la propia práctica, que resultó coherente con el marco teórico, es al mismo tiempo una evaluación de la coherencia interna en la formación de profesores, experiencia que sirvió para retroalimentar los procesos reflexivos de los formadores de formadores.

\section{Palabras clave}

Formación docente, modelo cognitivo de ciencia escolar, reflexión sobre la propia práctica (metarreflexión).

\section{Abstract}

In this paper, the production of educational practices in secondary school is analysed using as theoretical framework the Cognitive Model of School Science. The investigation was part of one of the authors' (Cabrera's) final thesis for the degree of science teaching; it was centred on the analysis of the researcher's and some other teachers' dassroom practices. The work permitted establishing the degree of coherence between the theoretical formulations and the practices that student teachers construct; at the same time, it permitted determining the analysis and reflection skills developed during their professional induction.

Teachers' analysis of their own practice, which was coherent with the theoretical framework, is at the same time an evaluation of the internal coherence in teachers' education; the experience was useful as feedback for the teacher educators' reflexive processes.

\section{Keywords}

Teacher education, cognitive model of school science, reflection on one's own practice (metareflexive processes). 


\section{Introducción}

\section{Objetivos}

En el presente trabajo relatamos una experiencia que es parte de la formación inicial del profesorado de Biología perteneciente a la Universidad de Buenos Aires, en Argentina, en la aual los futuros profesores realizan una tesis final de indagación acerca de algún aspecto de la institución escolar. En este contexto las observaciones de dase se transforman en un espacio curricular de análisis y reflexión de dichos aspectos.

En este proceso, se selecciona un contexto educativo y se define una perspectiva teórica a partir de la cual se desarrolla el análisis (Bonan et al., 2003). En el caso del trabajo que presentamos el análisis de las observaciones de dase se constituyó en un insumo para generar categońas que sirvieron para la reflexión sobre la propia práctica del investigador.

\section{Marco teórico}

Numerosos autores refieren la importancia de una formación inicial y continua del profesorado orientada a generar docentes cúticos y reflexivos, capacitados para producir buenas prácticas escolares y para revisar las prácticas instaladas en la escuela y producir nuevas prácticas y experiencias educativas para la enseñanza de las ciencias, que tengan como referente el aula real (Meinardi, 2007). Como señalan Copello y Sanmartí (2001) las acciones formadoras deben llevar a que el profesorado alcance una fundamentación teórica de su actuación y es imprescindible que el proceso de formación se vincule a una reflexión crítica tanto en relación con la forma actual de enseñar ciencias como a las posibles innovaciones planteadas. Por lo tanto, la formación profesional docente debería organizarse alrededor de una práctica que promueva en los futuros educadores y educadoras el desarrollo de procesos de reflexión de tipo metacognitivo que les permita reconocer, evaluar y decidir sobre la necesidad de reelaborar sus concepciones y su práctica (Meinardi, op. cit).

En este sentido, Perrenoud (2004) refiere que la formación inicial y permanente es uno de los "trampolines que permiten elevar el nivel de competencia de los practicantes", contribuyendo a aumentar sus saberes, transformar su identidad y su sentido ético. La autonomía y la responsabilidad de un profesional no se conciben sin una gran capacidad de reflexionar en la acción y sobre la acción. Por lo tanto, la figura del practicante reflexivo está en el centro del ejercicio de una profesión, por lo menos cuando la consideramos desde el punto de vista de la experiencia y de la inteligencia en el trabajo. Es necesario entonces, que la formación inicial prepare al futuro enseñante a "reflexionar sobre su práctica, centrarse en determinados temas, establecer modelos, ejercer la capacidad de observación, de análisis, de metacognición y de metacomunicación".

En acuerdo con estos supuestos, como parte final del trabajo de tesis de la formación del profesorado los estudiantes-futuros profesores de Biología de la Universidad de Buenos Aires realizan un trabajo de indagación acerca de alguna problemática relevante de la institución escolar. Se trata de un trabajo de reflexión sobre las prácticas de la enseñanza, de modo que los estudiantes se apropien del bagaje teórico que recibieron en su formación al realizar un análisis de algún aspecto de la realidad educativa. Para su ejeaución, los estudiantes deben seleccionar un contexto educativo sobre el que realizar las observaciones y definir una perspectiva teórica que les posibilite efectuar el análisis, situación que los conduce a autogestionar su propia práctica (Bonan et al., op. cit.).

En este contexto, se analizaron las dases y las actividades desarrolladas por tres docentes de escuela media (uno de ellos autor de este trabajo, es decir que realiza una reflexión sobre su propia práctica), teniendo en auenta el modelo cognitivo de ciencia escolar (MCCE) y otros autores 
aryos aportes son coherentes con dicho modelo. En función de esto se consideró la utilización de estrategias como la resolución de problemas, los experimentos de laboratorio y la indagación de las ideas previas de los alumnos, entre otras actividades. Este marco teórico ampliado permitió robustecer la fundamentación teórica en tomo al MCCE, y a la vez enriqueció el análisis de las actividades.

EI MCCE se postula como capaz de acercar la ciencia de los científicos a la ciencia de los alumnos, o ciencia escolar, definida como "una transposición didáctica del conocimiento cient́fico enudito, realizada de modo tal que posibilite a los alumnos acceder a un aprendizaje que les resulte significativo, que sea cercano a su realidad" (Izquierdo et al.,1999). La ciencia escolar comparte una fundamentación epistemológica con la ciencia de los científicos pero al mismo tiempo tiene características propias que la diferencian: es como la de los científicos en su aspecto esencial (el pensamiento teórico) pero autónoma en aspectos secundarios. Ni las experiencias, ni el lenguaje ni los hechos que configuran la ciencia escolar han de ser los de los cient́ficos.

La idea central que subyace en este modelo es la de la autonomía de la ciencia que se enseña y se aprende en la escuela, en relación a la selección y secuenciación de contenidos y al diseño de experimentos, actividades y problemas adecuados al contexto escolar a partir de lo que los alumnos son capaces de hacer reflexivamente. Se establece así una genuina actividad científica escolar que podría garantizar la aplicabilidad de los conocimientos escolares, lo cual requiere que los alumnos regulen sus propias acciones. Este es uno de los pilares del MCCE: la autonomía de los alumnos, que debe verse reflejada no sólo en las estrategias de enseñanza y actividades del docente, sino también en el modelo de evaluación puesto en juego.

Involucrar a los alumnos en la regulación de su propio aprendizaje garantizańa entonces su autonomía, opinando según sus propias ideas y adquiriendo el citerio necesario para discriminar ideas cient́ficas de las que no lo son. Asimismo, el docente debería tener en cuenta las ideas previas del alumnado, conceder importancia a los procesos de transposición didáctica e impulsar los procesos de metacognición y de autorregulación respecto de los procesos de construcción de los nuevos conocimientos (Izquierdo et al., op. cit.).

\section{Desarrollo}

Para llevar a cabo este trabajo se observaron dases de tres docentes de Biología (uno de ellos es el propio autor) y se analizaron las actividades planteadas en las mismas, seleccionándose aquellas que se consideraron más representativas y las que mayor información aportaron.

Teniendo en cuenta los fundamentos teóricos seleccionados se diseñó un instrumento de análisis que induyó la construcción de ciertas categorías e indicadores. Se denominó "categorías" a aquellas conceptualizaciones que intentan dar cuenta de lo observado en las clases. Estas fueron: Autonomía de los alumnos, Características generales de las actividades, Indagación de ideas previas, Tipos de problemas y Evaluación.

Para cada categoría se utilizaron indicadores que aportaron información y se constituyeron en insumos para el análisis. Para la categońa "Autonomía de los alumnos" se tuvieron en cuenta diversos indicadores como la existencia de instancias de reflexión individual o grupal en la resolución de una determinada actividad o problema, o las reflexiones de los alumnos al revisar sus propias producciones.

En la categoría "Características generales de las actividades" se analizó si el abordaje de los contenidos se daba en contextos próximos al de los alumnos, en conexión con sus intereses, como por ejemplo a través de cuestiones referidas a la vida cotidiana (Izquierdo et. al., op. cit). También 
se tuvo en cuenta si las actividades fomentaban: habilidades cognitivo-lingüísticas de tipo descriptivas y argumentativas, la formulación de preguntas, la generación de hipótesis, la contrastación de teońas o modelos, el diseño de experimentos por parte de los alumnos, la anticipación de resultados, entre otras. La puesta en juego de este tipo de habilidades les permitińa a los alumnos realizar tareas más complejas y de mayor calidad desde el punto de vista cognitivo.

Para la categoría "Indagación de Ideas Previas" se tomó como indicadores la existencia de genuinas indagaciones orientadas a conocer lo que el alumno cree o sabe sobre un determinado fenómeno, por ejemplo a través del uso de analogías, dibujos, o explicaciones de hechos puntuales orientados a poner en juego sus propios criterios y modelos explicativos. También se tuvo en arenta la existencia de actividades que promovieran el conflicto cognitivo y la desestabilización de dichas ideas previas, y actividades que funcionaran a modo de evaluación post-instruccional, que pudieran indicar el grado de persistencia de dichas concepciones en los alumnos, lo que permitińa regular el curso del proceso de enseñanza (Izquierdo et. al.,op. cit).

En cuanto a la categońa "Tipos de problemas" se analizó si los docentes planteaban actividades que constituyeran verdaderos problemas o si se trataba de ejercicios en los cuales los alumnos ya conocían las estrategias de resolución y además si eran de respuesta cerrada o abierta. También se tuvo en cuenta el nivel de investigación de las actividades: si se llevaban a cabo experimentos de laboratorio que representaban una verdadera "tarea investigativa", estando al servicio de la reflexión teórica o simplemente cumplían una función demostrativa o ilustrativa. Las actividades fomentarían una mayor utilización de estrategias científicas y de mayor calidad en la medida que dejen más grados de libertad para resolverlas en manos de los alumnos y admitan varias soluciones posibles.

Para la categoría "Evaluación" interesó ver si el docente realizaba evaluaciones diagnósticas y formativas que pudieran permitir conocer las dificultades de comprensión y regular el curso de los aprendizajes de sus alumnos o en cambio se trataba de evaluaciones orientadas solo a la calificación y a la acreditación.

Se analizó también el grado de coherencia entre el instrumento de evaluación, las estrategias de enseñanza y las actividades propuestas en las dases.

\section{Resultados}

En función de las categorías seleccionadas, en algunas dases y actividades propuestas por los docentes observados se notó escaso relevamiento de las ideas previas de los alumnos. Se promovieron instancias de resolución individual o grupal de ciertas actividades, pero las mismas no parecian estar orientadas hacia la reflexión.

En cuanto a la resolución de problemas, si bien eran de respuesta abierta, no parecían admitir múltiples resultados posibles ya que aparentemente dejaban poco margen para la formulación de hipótesis o para la puesta en juego de habilidades o estrategias cognitivo-lingǘísticas que permitieran la realización de planteamientos cualitativos fundamentales en la tarea científica.

Llegado a este punto, las categorías formuladas fueron utilizadas para revisar la planificación de las prácticas de residencia docente del propio investigador, el primer autor de este trabajo. Dichas prácticas habían sido planificadas en el trayecto de su formación docente, pero en un cuatrimestre anterior.

Se encontró un alto grado de coherencia con las prescripciones teóricas que los docentes de formación del profesorado utilizan para dar sentido a dichas prácticas. Por ejemplo, las actividades 
estaban orientadas a promover el conflicto cognitivo y a la desestabilización de las ideas previas y los modelos explicativos que las sustentan. Los problemas planteados eran de respuesta abierta y no aceptaban una única estrategia de resolución. También se encontraron instancias que promovían la reflexión individual y grupal. En cuanto a las actividades de evaluación, la utilización de un instrumento diseñado a partir de las ideas previas de los alumnos podría permitir al docente detectar la persistencia de ciertas concepciones así como difiaultades de comprensión, pudiendo dar lugar a la toma de conciencia por parte de los alumnos de la existencia de tales representaciones, lo cual fomentaría la metacognición y la autorregulación de sus aprendizajes.

\section{Conclusiones}

La reflexión sobre esta experiencia se constituye en un ejercicio metacognitivo respecto del impacto de las prescripciones teóricas sobre las prácticas de los futuros profesores.

Tal como señalan Copello y Sanmartí (2001) "la metacognición actúa posibilitando la formación de esquemas mentales o representaciones dinámicas que surgen como producto de las experiencias resultantes de las actividades del sujeto sobre la realidad, y su mecanismo principal es la reflexión; cada acto metacognitivo tiene lugar a través de un proceso de reflexión".

Numerosos autores coinciden en afirmar la importancia de formar docentes reflexivos, autónomos y responsables, capaces revisar su propia práctica y de generar nuevas experiencias educativas. En palabras de Perrenoud (2004), "nada de todo ello se adquiere como por arte de magia ni sencillamente porque uno tenga éxitos y fracasos en su trabajo. Todo el mundo reflexiona para actuar, durante y después de la acción, sin que esta reflexión provoque sistemáticamente aprendizajes. El reto estriba en proporcionar a la vez actitudes, habitus, saber hacer, en el método y en las posturas reflexivas". Por lo tanto, es fundamental promover el desarrollo de habilidades metacognitivas desde la formación inicial creando "los lugares para el análisis de la práctica, de mestizaje de las aportaciones y de reflexión sobre la forma cómo pensamos, decidimos, comunicamos y reaccionamos en una dase En pocas palabras, sólo conseguiremos formar practicantes reflexivos a través de una práctica reflexiva, en virtud de "aprender a hacer lo que no se sabe hacer, haciéndolo".

De allí que las docentes de formación del profesorado -y co autoras de este trabajoconsideramos que el trabajo de análisis y reflexión de las prácticas de enseñanza en las escuelas contribuye en la construcción de una didáctica fundamentada, pero al mismo tiempo resaltamos que cuando este trabajo se realiza sobre la propia práctica del docente en formación, como la que realiza el primer autor y presentamos aquí, es una estrategia de metarreflexión que contribuye también a los procesos formativos de los y las formadores de formadores.

\section{Bibliografía}

Bonan, L; Galagovski, L; Adúriz Bravo, A.; Revel Chion, A.; González Galli, L; Meinardi, E. (2003). El análisis de la realidad del aula a través de las Observaciones de Clase. III Congreso Nacional y I Intemacional de Investigación Educativa. Cipolletti, Argentina.

Copello Levy, M. I.; Sanmartí Puig, N. (2001). Fundamentos de un modelo de formación permanente del profesorado de ciencias centrado en la reflexión dialógica sobre las concepciones y las prácticas. Enseñanza de las Ciencias, 19 (2), 269-283.

Izquierdo, M.; Espinet, M.; Garća, M. P.; Pujol, R. M; Sanmartí, N. (1999). Caracterizadón y fundamentación de la ciencia escolar. Enseñanza de las ciencias, Número extra, 79-91. 
Meinardi, E. (2007). Reflexiones sobre la formación inicial de los profesores de Biología. Revista de Educación en Biología, 10 (2), 48-54.

Perrenoud, P. (2004). Desarrollar la práctica reflexiva en el oficio de enseñar profesionalización y razón pedagógica. Barcelona: Graó. 